\title{
Protective effects of beef decoction rich in carnosine on cerebral ischemia injury by permanent middle cerebral artery occlusion in rats
}

\author{
AI-HONG WANG ${ }^{1}$, QIAN MA ${ }^{1}$, XIN WANG $^{1}$ and GUI-HUA XU ${ }^{2}$ \\ Departments of ${ }^{1}$ Basic Nursing and ${ }^{2}$ Chinese Medicine Nursing, Institute of Nursing, \\ Nanjing University of Chinese Medicine, Nanjing, Jiangsu 210023, P.R. China \\ Received March 18, 2016; Accepted February 24, 2017
}

DOI: $10.3892 /$ etm.2017.5524

\begin{abstract}
Inflammation has a role in the cerebral injury induced by ischemia and the present study aimed to determine the mechanism of the protective effect of beef decoction (BD) with carnosine against it. A rat model of permanent middle cerebral artery occlusion was established using a suture method in the vehicle and each of the BD groups. In experiment 1, 72 Sprague Dawley (SD) rats were randomly divided into three groups: Sham, vehicle and BD-treated group. Rats in the BD group were given $600 \mathrm{mg} / \mathrm{kg} \mathrm{BD}$ by oral gavage for 1, 3 and 7 days. The sham and vehicle group rats received an equivalent amount of normal saline. In experiment 2, $60 \mathrm{SD}$ rats were randomly divided into six groups: Sham-operated I, sham-operated II, vehicle, low-dose BD, medium-dose $\mathrm{BD}$ and high-dose BD group. Rats in the low-, medium- and high-dose BD groups were given BD at the dose of 200, 400 and $600 \mathrm{mg} / \mathrm{kg}$, respectively, by oral gavage for 7 days. Rats in the sham-operated II group were given $600 \mathrm{mg} / \mathrm{kg} \mathrm{BD}$. Rats in the sham-operated I group and vehicle group were given the same volume of normal saline by oral gavage. The body weight, neurological deficits and infarct volume were recorded at 1, 3 and 7 days after the operation. Furthermore, the effect of different doses of BD on interleukin-6 (IL-6), tumor necrosis factor- $\alpha$ (TNF- $\alpha)$, interferon- $\gamma($ IFN- $\gamma)$ and interleukin-4 (IL-4) levels in peripheral blood was measured at 7 days. BD-treated rats showed less neurological deficits and a smaller infarct volume at 7 days. BD at 400 and $600 \mathrm{mg} / \mathrm{kg}$ significantly decreased the infarct volume in rats. At $600 \mathrm{mg} / \mathrm{kg} \mathrm{BD}$, a decline in IL-6, TNF- $\alpha$, IFN- $\gamma$ and an increase in IL-4 expression was observed in the BD groups,
\end{abstract}

Correspondence to: Dr Gui-Hua Xu, Department of Chinese Medicine Nursing, Institute of Nursing, Nanjing University of Chinese Medicine, 138 Xianlin Road, Nanjing, Jiangsu 210023, P.R. China

E-mail: xgh_88@126.com

Key words: beef decoction, carnosine, cerebral ischemia, cytokines, inflammation while no difference in body weight and neurological dysfunction was detected. In conclusion, BD is a neuroprotective agent that may be used as a supplement treatment of ischemic stroke.

\section{Introduction}

Stroke is the second leading cause of mortality and the third leading cause of disability worldwide. Over the last two decades, a $26 \%$ increase in stroke-associated deaths with $19 \%$ disability-adjusted life years has been observed (1,2). By 2020, the overall cost for stroke has been predicted to have increased by $6.2 \%$ (3), leading to a major social, economic and medical burden in the near future. Ischemic stroke is the predominant stroke subtype (4), accounting for 85-90\% of stroke events (5). Cerebral ischemia results from a transient or permanent reduction of blood supply, causing insufficient oxygen and glucose delivery to support cellular homeostasis. During this course, the pathophysiological processes are complex and extensive and include excitotoxicity, acid toxicity and ionic imbalance, peri-infarct depolarization, oxidative and nitrative stress, inflammation and apoptosis (6). In recent years, is increasing evidence has shown that the inflammatory response has a pivotal role in cerebral ischemia-induced brain injury. It is thought that the anti-inflammatory response and alleviation of brain damage secondary to ischemia are promising approaches for post-stroke therapy (7).

Immune and brain cells, including astrocytes, microglia or endothelia, have been found to be activated by ischemic stroke. These cells in turn become immunologically reactive and interact with each other to produce cytokines and adhesion molecules $(8,9)$. Cytokines can be divided into pro-inflammatory and anti-inflammatory cytokines according to their roles in the inflammatory process (10), and trigger the inflammation due to their imbalance following cerebral ischemia. Regulating the synthesis and secretion of pro-and anti-inflammatory cytokines is key to controlling late cerebral ischemic inflammation (11).

Carnosine ( $\beta$-alanyl-L-histidine) is an endogenous dipeptide isolated from natural material, which occurs in the brain, heart and skeletal muscle, and easily migrates into the central nervous system from the periphery (12). Earlier studies have shown that carnosine possesses various neuroprotective 
properties, such as anti-oxidative $(13,14)$, anti-glutamatergic excitotoxic (15) and anti-apoptotic activity (16) in models of cerebral ischemia. A previous study by our group showed that carnosine regulated immune responses and reduced brain damage in a rat model of ischemia (17). Due to its beneficial effects, carnosine is considered as an attractive therapeutic candidate for ischemic stroke damage. However, at present, carnosine is synthetically produced, which is expensive. In addition, the synthesis process leads to the production of hydrazine, which is hazardous. Therefore, a better natural source of carnosine is required, which has become an important research focus in recent years.

Beef decoction (BD) was previously prepared by our group based on the theory of 'Medicine Food Homology'. An amino acid composition detection method was used to quantify the carnosine content in the $\mathrm{BD}$, and the results showed that the amino acids in carnosine account for $63.05 \%$ of the total amino acid content. A previous study by our group showed that $\mathrm{BD}$ protected the brain from the damage caused by permanent middle cerebral artery occlusion (pMCAO), and that the protective effect was due to its anti-inflammatory properties (18). In the present study, BD treatment in pMCAO rats was explored with improved duration and dosage levels. In addition, the present study analyzed whether BD affects the levels of pro- and anti-inflammatory factors, which are deregulated in response to ischemic stroke.

\section{Materials and methods}

Preparation of $B D$. BD was prepared by the previously reported method (18). Exactly $1 \mathrm{~kg}$ of beef ham was cut into $2-\mathrm{cm}^{3}$ cubes and minced using a meat grinder. The minced beef was boiled in water (minced beef/water ratio, 1:2) in a pot for regulated by a power regulator for a total of $12 \mathrm{~h}$. Subsequently, oil was skimmed off the resulting BD, the suspension was strained through a colander and further filtered with gauze. BD was concentrated using a rotary evaporator (YaRong Biochemistry Instrument Factory, Shanghai, China) and the final concentrated BD had a volume of $60 \mathrm{ml}$. It contained $36.34 \mathrm{mg} / \mathrm{kg}$ carnosine, which was detected by an S-433D amino acid analyzer (Sykam, Eresing, Germany).

Experimental animals. A total of 120 male Sprague Dawley (SD) rats (age, 10-12 weeks; weight, 280-320 g) were supplied by the Experimental Animal Center in Nanjing University of Chinese Medicine (Nanjing, China). The rats were bred in-house under standard laboratory conditions with controlled temperature $\left(21 \pm 3^{\circ} \mathrm{C}\right)$, relative humidity of $55-70 \%$ and a $12-\mathrm{h}$ light/dark cycle. Water and food were available ad libitum. The experimental study protocol was approved by the Ethics Committee of the Laboratory Animal Center of Nanjing University of Chinese Medicine [ACU-39 (20141226)]. All procedures involving animal care complied with the China National Institutes of Health Guidelines for the Care and Use of Laboratory Animals.

Rat model of permanent focal cerebral ischemia. To mimic cerebral ischemia, a rat model of pMCAO was generated. The rats were anesthetized using $10 \%$ chloral hydrate (350 mg/kg intraperitoneal; Sinopharm Chemical Reagent
Co., Ltd, Shanghai, China). The right common carotid artery (CCA), external carotid artery (ECA) and internal carotid artery (ICA) were exposed and carefully isolated. The distal end of the ECA and the CCA were ligated with a 6-0 silk suture, another silk suture was placed loosely around the ICA and a microsurgical clip was placed on the ICA. A nylon monofilament (40 $\mathrm{mm}$ in length and $0.26 \mathrm{~mm}$ in diameter; Sunbio Biotech Ltd., Beijing, China), with a smooth and rounded tip, was introduced into the ICA through a small puncture in the CCA and advanced into the MCA via the ICA $(18-20 \mathrm{~mm}$, as measured from the start of the carotid bifurcation), until a slight resistance was felt. The silk suture around the ICA was tightened to secure the intraluminal nylon suture and prevent bleeding. At this point, the origin of the MCA was blocked by the intraluminal filament. The sham-operated rats were subjected to similar surgical procedures as the pMCAO rats, except that the filament was not inserted. Two $h$ after the induction of ischemia, the model was evaluated according to Longa's method (19), and the neurological deficits were scored on a five-point scale: 0 , no apparent neurological deficit; 1 , failure to fully extend the contralateral forepaw; 2, circling to the left side; 3 , falling towards the left side; and 4, inability to walk. The animals which received a score in the range of 1-3 were used for further treatment studies.

$B D$ treatment. The present study involved two stages of BD treatments, with the BD dosage at different time intervals in the first experiment and different BD doses in the second experiment. In the first experiment, 72 rats were randomly divided into three groups: Sham, vehicle and BD-treated group ( $n=8$ per group and per time-point). The BD group rats were given a standard amount of BD $(600 \mathrm{mg} / \mathrm{kg})$ by oral gavage for 1, 3 and 7 days, twice daily. The sham and vehicle group rats received an equivalent amount of sterilized normal saline. In the second experiment, $60 \mathrm{SD}$ rats were randomly divided into six groups: Sham-operated I, sham-operated II, vehicle, low-dose BD, medium-dose BD and high-dose BD group. The rats in the low-, medium- and high-dose BD groups were given $\mathrm{BD}$ at a dose of $200,400,600 \mathrm{mg} / \mathrm{kg}$ by oral gavage, respectively for 7 days. Assuming a body weight of $300 \mathrm{~g}$, rats in the high-dose BD group should be administered $180 \mathrm{mg}$ carnosine by oral gavage every day; the carnosine content in BD was $36 \mathrm{mg} / \mathrm{ml}$ (18). Therefore, the rats in the high-dose BD group were given $\sim 5 \mathrm{ml} \mathrm{BD}$ by oral gavage daily. In the same way, rats in the medium- and low-dose BD groups were given $~ 3.33$ and $1.67 \mathrm{ml} \mathrm{BD}$ by oral gavage daily, respectively. In order to keep experimental uniformity, rats in the low- and medium-dose BD groups were given 200 and $400 \mathrm{mg} / \mathrm{kg} \mathrm{BD}$, respectively, by oral gavage, which was diluted with $5 \mathrm{ml}$ distilled water. Sham-operated I group and vehicle group were given $5 \mathrm{ml}$ normal saline by oral gavage. The rats in the sham-operated II group were given $600 \mathrm{mg} / \mathrm{kg}$ BD by oral gavage. The rats were given $\mathrm{BD}$ for the first time at $2 \mathrm{~h}$ after the onset of ischemia, followed by $2.5 \mathrm{ml} \mathrm{BD}$ twice daily for 7 days.

Monitoring of general condition, bodyweight and neurological score. During the first experiment, the general condition of the rats, such as their activity and color of their coat was observed. The body weight of rats from each group was determined at various time points (days 1, 3 and 7 in the first experiment 


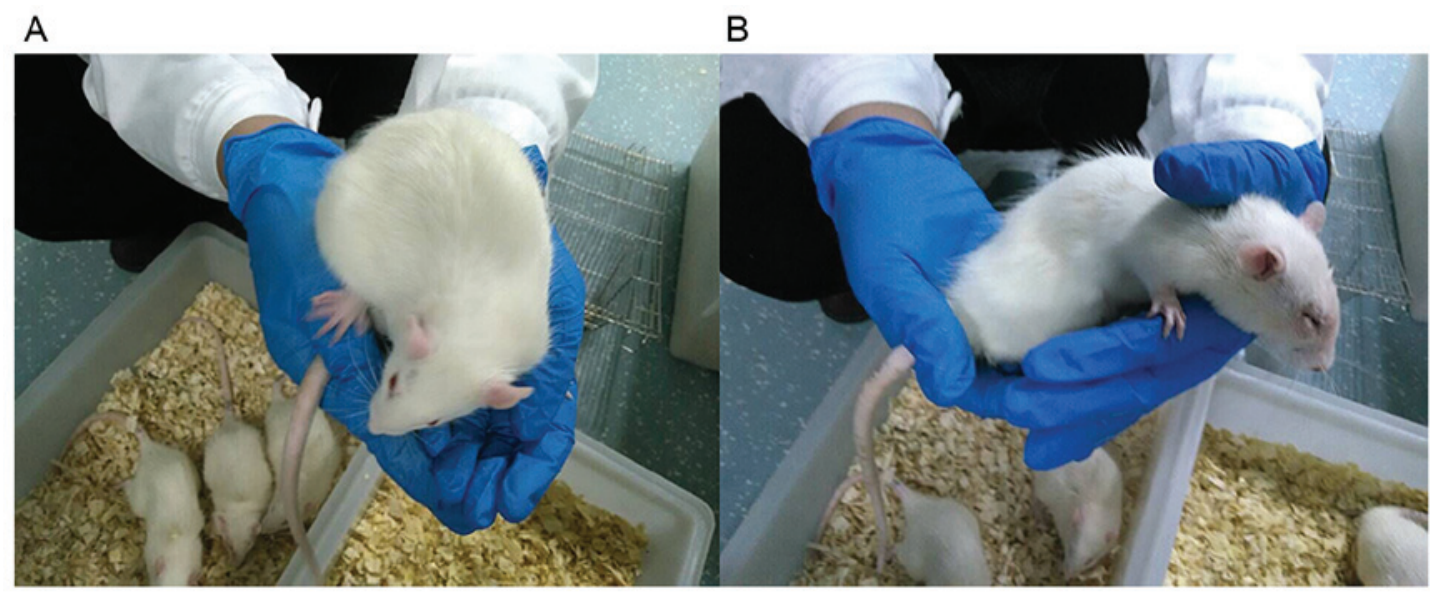

Figure 1. General condition of the rats in (A) the beef decoction-treated group and (B) the vehicle group.

and day 7 in the second experiment). At these time points, the neurological deficits of rats were assessed using the Zea longa test (19) and expressed as neurological grade score.

Quantification of infarct area. The infarct volume of the rats from each group was determined at various time-points (days 1, 3 and 7 in the first experiment and day 7 in the second experiment). Rats ( $n=6$ per group) were euthanized under anesthesia, and the brain was immediately removed and cut into five coronal sections $(2 \mathrm{~mm})$, which were immediately incubated in $2 \%$ 2,3,5-triphenyltetrazolium chloride solution (Sigma-Aldrich; Merck Millipore KGaA, Darmstadt, Germany) at $37^{\circ} \mathrm{C}$ for $30 \mathrm{~min}$ in the dark. Following immersion in $10 \%$ formalin overnight, images of the stained slices were captured using a digital camera. The total infarct volume of the right hemisphere (ischemic side) and the total volume of the left hemispheres in each section was calculated using the Image-Pro Plus 6.0 software (Media Cybernetics, Rockville, MD, USA) using the following formula: Infarct area $(\%)=$ total infarct area of the right hemisphere/total area of the left hemispheres x100\% (20).

Histology. In the second experiment, 4 rats were randomly selected from each group, respectively, after pMCAO, anesthetized intraperitoneally with $10 \%$ chloral hydrate $(350 \mathrm{mg} / \mathrm{kg}$; Sinopharm Chemical Reagent Co., Ltd.) and placed on an operating board with supine position. The heart was exposed, and the cardiac apex was inserted through the needle. The right atrial appendage was cut open, perfused with $500 \mathrm{ml}$ normal saline solution for $30 \mathrm{~min}$ followed by $4 \%$ paraformaldehyde at room temperature. Following this perfusion fixation, brains were removed immediately and immersed in $4 \%$ paraformaldehyde at $4^{\circ} \mathrm{C}$ for $48 \mathrm{~h}$, sequentially processed for paraffin embedding and sectioned into $5-\mu \mathrm{m}$ thick slices. The sections were placed in hematoxylin for $20 \mathrm{~min}$ at room temperature, washed three times with water, treated with hydrochloric acid in alcohol for color separation, and then washed three times with water. Subsequently, the sections were stained with eosin for $1 \mathrm{~min}$ at room temperature, washed 3-4 times, and dehydrated with 80 and $95 \%$ alcohol (once each), and $100 \%$ alcohol twice. Following this, the sections were permeabilized twice with xylene, mounted with neutral balsam, and observed using a light microscope (magnification x400; Nikon Eclipse
TE2000-U; Nikon, Tokyo, Japan). Cortical penumbra areas were selected for morphological observation and cerebral injury assessment.

ELISA. After the second experiment, the protein levels of interleukin (IL)-6, tumor necrosis factor (TNF)- $\alpha$, interferon (IFN) $-\gamma$ and IL-4 in the peripheral blood of rats from each group $(n=8)$ were detected. The rats were anesthetized by administering $10 \%$ chloral hydrate $(350 \mathrm{mg} / \mathrm{kg})$ intraperitoneally and blood was drawn from the left ventricle. The blood specimens were incubated in $37^{\circ} \mathrm{C}$ for $30 \mathrm{~min}$ and the serum was collected by centrifuging the samples at $587 \mathrm{x} \mathrm{g}$ for $10 \mathrm{~min}$. Levels of IL-6, TNF- $\alpha$, IFN- $\gamma$ and IL- 4 were measured in the serum using ELISA kits (Nanjing Jiancheng Biological Engineering Institute, Nanjing, China) according to the manufacturer's recommendation. ELISA samples $(n=8$ for each group) were measured using a microplate reader (Perkin Elmer, Waltham, MA, USA) at $450 \mathrm{~nm}$. Cytokine concentrations were calculated using Table Curve 3D Software (SPSS Inc., Chicago, IL, USA).

Statistical analysis. The standard software package SPSS 17.0 (SPSS Inc.) was used for statistical analyses. All data were presented as the mean \pm standard deviation. One-way analysis of variance was employed when comparing multiple groups and a least significant difference post hoc test was used for comparing two groups. $\mathrm{P}<0.05$ was considered to indicate a statistically significant difference.

\section{Results}

$B D$ improves the overall condition of rats after $p M C A O$. On the first day after pMCAO, rats appeared depressive, lethargic, tired and lackluster, and were slow in their responses and movement with reduced food intake. After intragastric administration of BD for 7 days, a significant change in their behavioral pattern was observed with rats being active and animate, normal food intake and vivid hair color (Fig. 1A) compared to that of vehicle-treated rats (Fig. 1B).

Body weight in the experimental groups. In the first experiment, a significant reduction in body weight was observed 


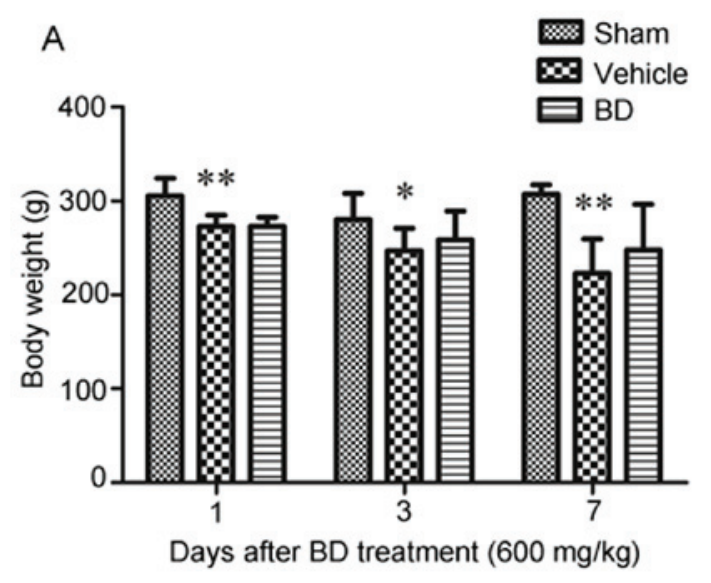

B

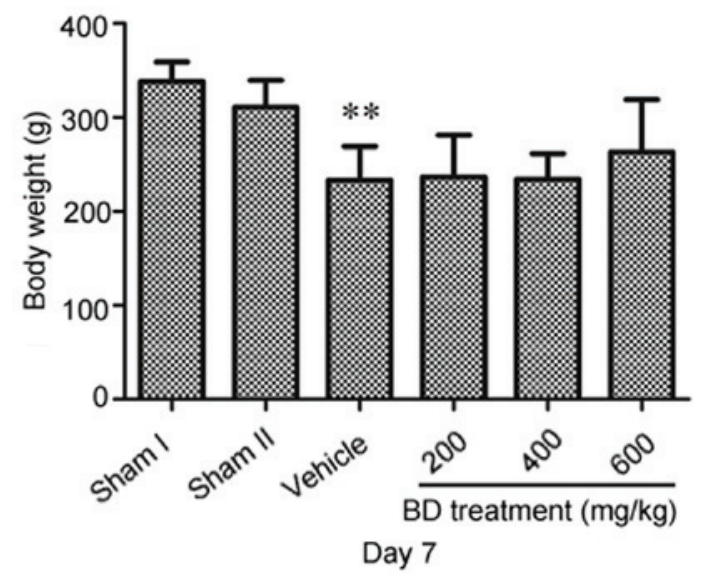

Figure 2. Evaluation of body weight over the course of the experiment. (A) Body weight at 1, 3 and 7 days after treatment. (B) Body weight in the different experimental groups at day $7 .{ }^{*} \mathrm{P}<0.05 ;{ }^{* *} \mathrm{P}<0.01$ vs. the sham-operated group. Values are expressed as the mean \pm standard deviation. BD, beef decoction.

in the vehicle-treated group compared to the sham group at 1,3 and 7 days $(\mathrm{P}<0.01, \mathrm{P}<0.05$ and $\mathrm{P}<0.01$, respectively; Fig. 2A). The decrease in body weight was less pronounced in BD-treated rats compared to that in the vehicle group at 3 and 7 days, but the difference between the two groups was not significant $(\mathrm{P}>0.05$; Fig. $2 \mathrm{~A})$. In the second experiment, the body weight of the rats in different groups was evaluated at day 7 . While no significant difference in body weight was observed between sham I and sham II groups ( $\mathrm{P}>0.05$; Fig. 2B), rats in vehicle-treated group showed a significant decline in body weight compared to that in the sham groups $(\mathrm{P}<0.01$; Fig. 2B). No significant differences in body weight were observed between the vehicle and BD-treated groups (P>0.05; Fig. 2B).

$B D$ treatment reduces neurological dysfunction in rats after cerebral ischemia. In the first experiment of the study, no neurological deficits were present in the sham group, but focal cerebral ischemia caused a prominent impairment of motor performance in rats, and severe neurological deficits were observed in the rats in the vehicle compared to those in the sham group at 1,3 and 7 days after treatment $(\mathrm{P}<0.01)$. A

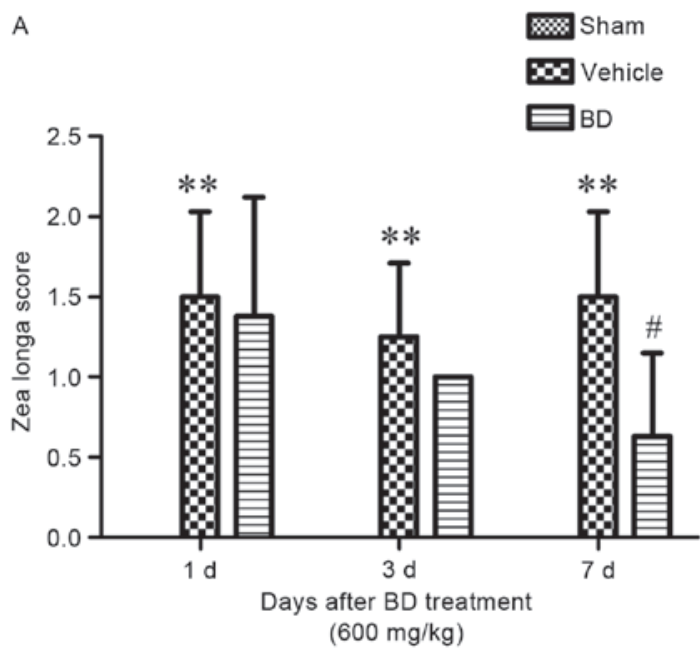

B

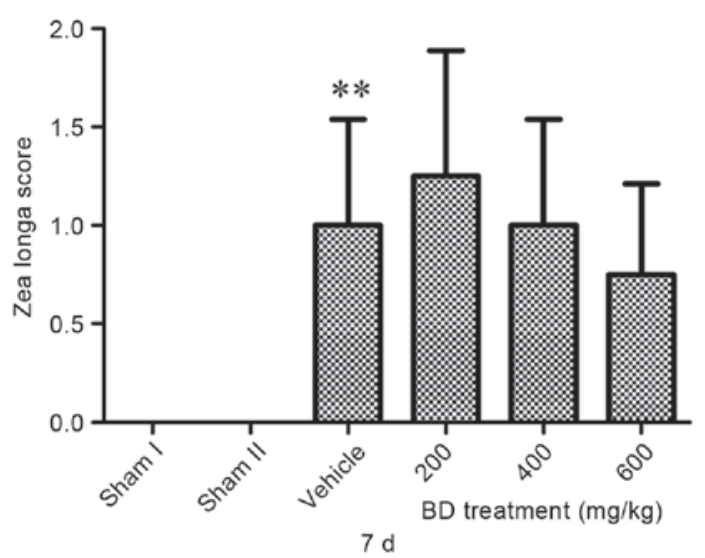

Figure 3. Evaluation of neurological deficits after treatment. (A) Effect of BD treatment on the Zea longa score at 1, 3 and 7 days after treatment. (B) Zea longa scores in the different experimental groups at 7 days after treatment. ${ }^{* * *} \mathrm{P}<0.01$ vs. the sham-operated group; ${ }^{~} \mathrm{P}<0.05$ vs. the vehicle-treated group. Values are expressed as the mean \pm standard deviation. BD, beef decoction; d, days.

significant reduction in the neurological scores was observed in the BD treatment group compared to that in the vehicle group after 7 days $(\mathrm{P}<0.05 ;$ Fig. $3 \mathrm{~A})$. In the second stage, no dysfunction was observed in the sham-operated rats (sham I and II groups). The high-dose BD group receiving $600 \mathrm{mg} / \mathrm{kg}$ BD showed a decrease in the Zea longa score compared with that in the other groups, which was, however, not statistically significant ( $\mathrm{P}>0.05$; Fig. 3B).

$B D$ treatment attenuates cerebral infarction. In the sham group, no cerebral injury was present, while an infarct area was observed in the right cortex and striatum of the vehicle group. In the first experiment, a smaller infarct volume was observed in the rats treated with $600 \mathrm{mg} / \mathrm{kg}$ BD for 7 days compared to that in the vehicle group $(\mathrm{P}<0.05$; Fig. 4$)$. In the second experiment, treatment with $\mathrm{BD}$ at 400 and $600 \mathrm{mg} / \mathrm{kg}$ markedly reduced the infarct volume in the BD group compared to that in the vehicle group $(\mathrm{P}<0.01)$. The infarct volumes in the vehicle group and the 200,400 and $600 \mathrm{mg} / \mathrm{kg}$ BD groups were $40.82 \pm 4.86,33.95 \pm 3.62,21.76 \pm 3.54$ and $21.19 \pm 5.50 \%$, respectively. After treatment with 200,400 and $600 \mathrm{mg} / \mathrm{kg}$ of $\mathrm{BD}$, the infarct volume was reduced by $16.83,46.69$ and 


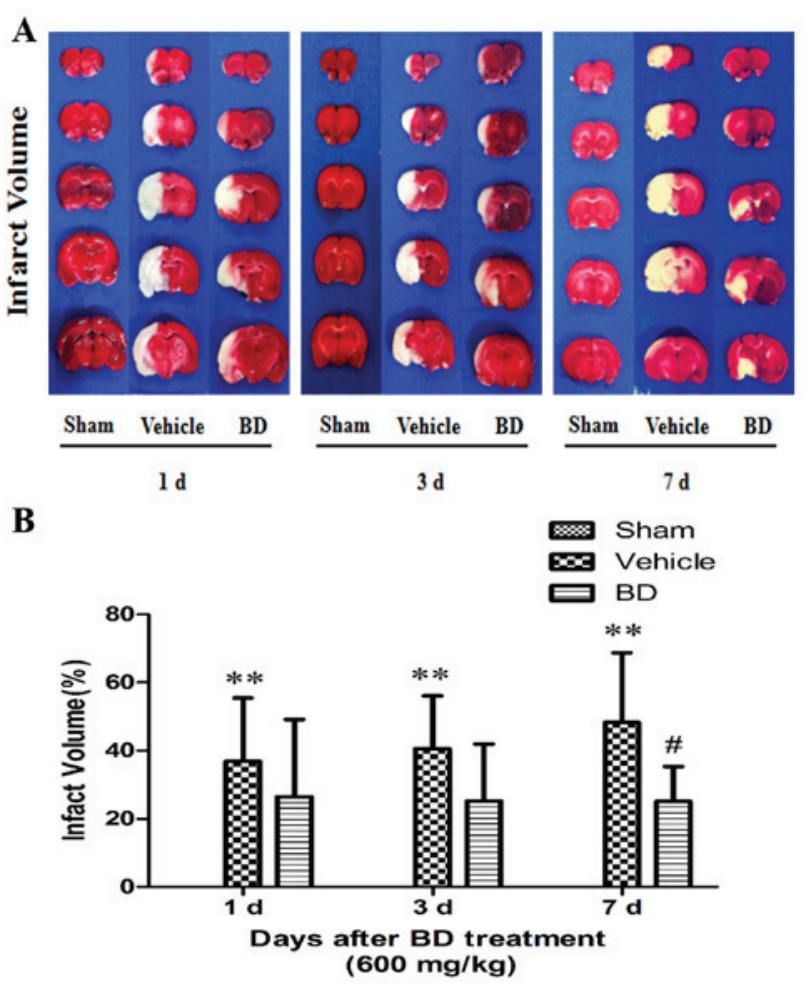

Figure 4. Infarct volume in the brains of rats treated with BD $(600 \mathrm{mg} / \mathrm{kg})$ or saline as well as sham-operated rats. (A) 2,3,5-Triphenyltetrazolium chloride staining of cerebral slices in each group and 1, 3 and 7 days after treatment. (B) Quantified infarct volume in all groups at all time-points. ${ }^{* *} \mathrm{P}<0.01$ vs. the sham group; ${ }^{\prime} \mathrm{P}<0.05$ vs. the vehicle group. Values are expressed as the mean \pm standard deviation. $\mathrm{BD}$, beef decoction; d, days.

$48.09 \%$, respectively, compared to that in the vehicle group. At 600 and $400 \mathrm{mg} / \mathrm{kg}$, BD significantly reduced the infarction area compared to that in the vehicle group $(\mathrm{P}<0.01$; Fig. 5).

Effect of BD on histological changes. Following hematoxylin and eosin staining, cortical penumbra areas were observed (Fig. 6A-F). Compared with the vehicle-treated sham I group, administration of BD $(600 \mathrm{mg} / \mathrm{kg})$ in the sham II group appeared to have no side effects (Fig. 6A and B). In the vehicle group, damaged neurons exhibited pyknosis and anachromasis of nuclei and severe cellular edema was present in cortical penumbra areas (Fig. 6C). Administration of BD $(200 \mathrm{mg} / \mathrm{kg})$ did not significantly attenuate this damage, while $\mathrm{BD}$ at higher doses of 400 and $600 \mathrm{mg} / \mathrm{kg}$ markedly protected the neurons from ischemic injury (Fig. 6D-F).

$B D$ treatment regulates cytokine expression of $I L-6, T N F-\alpha$, $I F N-\gamma$ and IL-4 after ischemia. The levels of pro- and anti-inflammatory cytokines, including IL-6, TNF- $\alpha$, IFN- $\gamma$ and IL-4 were markedly elevated in the vehicle group compared to those in the sham groups $(\mathrm{P}<0.01$ or $\mathrm{P}<0.05$; Fig. 7). Treatment with $600 \mathrm{mg} / \mathrm{kg}$ BD significantly downregulated IL-6 expression compared to that in the vehicle group (5.66 \pm 1.79 vs. $9.78 \pm 1.31 \mathrm{ng} / \mathrm{l} ; \mathrm{P}<0.05$; Fig. 7A). A significant decline in TNF- $\alpha$ was observed in the 400 and $600 \mathrm{mg} / \mathrm{kg}$ BD groups compared to that in the vehicle group $(96.77 \pm 47.72$ and $96.12 \pm 31.30 \mathrm{ng} / \mathrm{l}$, respectively, vs. $128.82 \pm 18.88 \mathrm{ng} / \mathrm{l}$; $\mathrm{P}<0.01$; Fig. 7B). Downregulation of IFN $-\gamma$ in the BD group

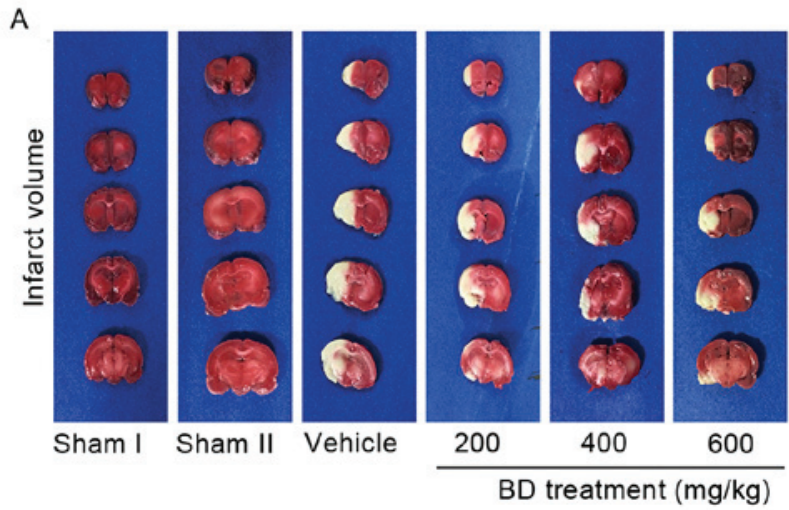

B

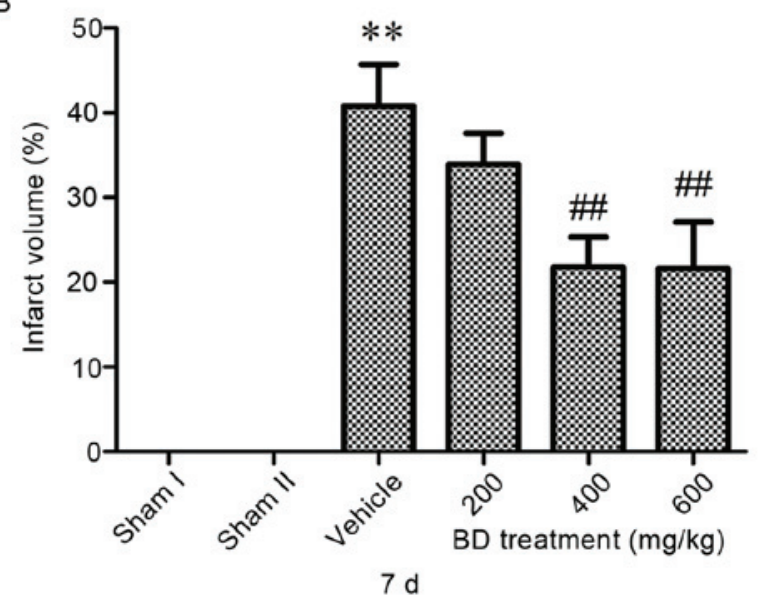

Figure 5. Infarct volume in the brains of rats treated with different doses of BD. (A) 2,3,5-Triphenyltetrazolium chloride staining of cerebral slices in each group. (B) Effect of various doses of BD on the infarct volume at 7 days after treatment. The sham I group received normal saline, while the sham II group was treated with $600 \mathrm{mg} / \mathrm{kg} \mathrm{BD} .{ }^{* *} \mathrm{P}<0.01$ vs. the sham groups; ${ }^{\# \#} \mathrm{P}<0.01$ vs. the vehicle group. Values are expressed as the mean \pm standard deviation. BD, beef decoction; d, days.

was observed at a dose of $600 \mathrm{mg} / \mathrm{kg}$ BD $(456.33 \pm 97.88 \mathrm{ng} / \mathrm{l}$; $\mathrm{P}<0.01)$, which was more significant than at doses of 200 and $400 \mathrm{mg} / \mathrm{kg}$ BD $(489.33 \pm 74.25$ and $515.77 \pm 47.73 \mathrm{ng} / \mathrm{l}$, respectively; Fig. 7C). By contrast, treatment with $600 \mathrm{mg} / \mathrm{kg}$ BD upregulated IL-4 expression (354.71 $\pm 58.33 \mathrm{ng} / \mathrm{l})$ compared to that in the vehicle group (294.54 $\pm 34.63 \mathrm{ng} / \mathrm{l}$; $\mathrm{P}<0.05$; Fig. 7D). Overall, treatment with BD at the dose of $600 \mathrm{mg} / \mathrm{kg}$ caused a decline in the levels of IL- 6 , TNF- $\alpha$, IFN- $\gamma$ and an increase in the levels of IL-4 in rats with cerebral ischemia.

\section{Discussion}

Carnosine has been proposed as an attractive therapeutic candidate for ischemic stroke. It is an endogenous dipeptide composed of alanine and histidine, which is found in numerous bodily tissues and also in the central nervous system (21). Exogenous carnosine has a neuroprotective role in the brain of pMCAO rats by migrating through the blood-brain barrier $(22,23)$. Previous in vitro and in vivo studies have indicated that carnosine exerts various neuroprotective effects in cerebral ischemia, such as anti-oxidant (24), anti-inflammatory (25), anti-glutamatergic excitotoxic (15), immune-regulatory (17) and anti-apoptotic effects $(16,26)$. The most commonly used method to synthesize carnosine is by chemical synthesis, which mainly uses $\beta$-alanine 

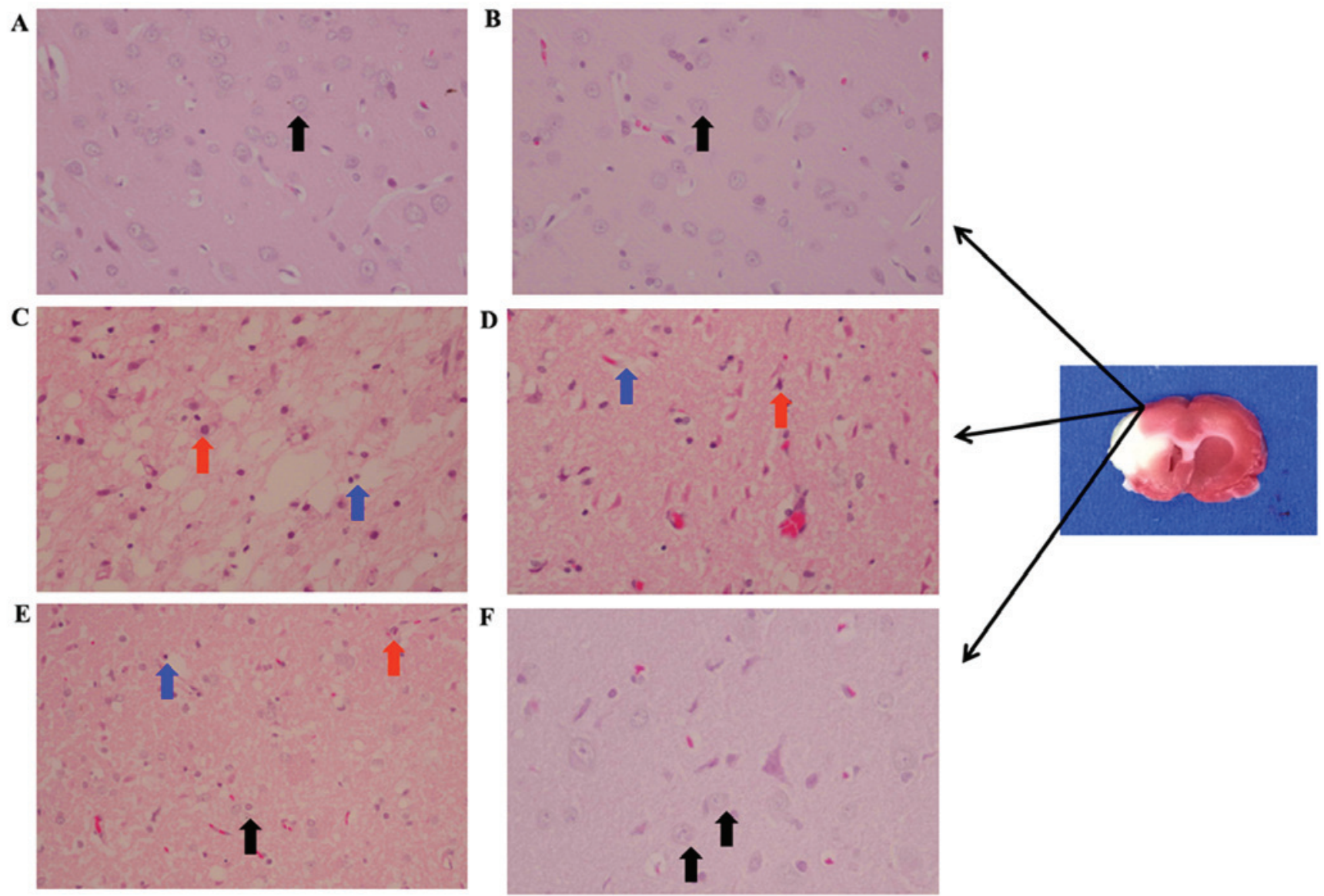

Figure 6. Histological examination of cortical penumbra areas in the brains of rats. Representative sections stained by hematoxylin and eosin from (A) sham I group and (B) sham II group. Compared with (C) the vehicle-treated group, a concentration-dependent improvement regarding condensed nuclei (orange arrows) and cellular edema (blue arrows) in cortical penumbra cells was observed in (D) the BD $200 \mathrm{mg} / \mathrm{kg}$ group, (E) BD $400 \mathrm{mg} / \mathrm{kg}$ group and (F) BD $600 \mathrm{mg} / \mathrm{kg}$ group. The sham I group received normal saline, while the sham II group was treated with $600 \mathrm{mg} / \mathrm{kg} \mathrm{BD}$. BD, beef decoction. Black arrows indicate normal cells.

or $\beta$-alanine precursor and L-histidine as the starting material; however, this method is time-consuming with low yield and high cost $(27,28)$. Extraction of meat (e.g., beef or chicken) with boiling water may be a better method of obtaining carnosine, since it is a water-soluble dipeptide. In a previously published procedure, meat was homogenized and centrifuged, and the supernatant extracted contained carnosine; however, the method was associated with certain complications (29). A previous study by our group reported on the development of a homemade beef decoction containing nearly $2.64 \mathrm{mg} / \mathrm{ml}$ carnosine; this method was safe and less expensive than the previous one, and was applied in the present study (18). The sham I and sham II groups treated with BD showed no significant difference in body weight, neurological scores, infarct volumes and level of cytokines $(\mathrm{P}>0.05)$, indicating that it had no side effects in rats.

Beef, particularly lean beef, is an important source of nutrients in the diet. Consumption of lean beef with low fat allows for higher intake of vitamins $\mathrm{A}, \mathrm{C}, \mathrm{B}_{6}$ and $\mathrm{B}_{12}$, niacin, phosphorus, magnesium, iron, zinc, potassium and protein, and lower intake of total energy, total fat, saturated fatty acid, mono-unsaturated fatty acids and total carbohydrates (30-32). Cholesterol-raising fatty acids and saturated fatty acid (SFA) have been associated with cardiovascular diseases. Only one-third of the fatty acids in beef are cholesterol-raising fatty acids and one-third of the SFA in beef consists of stearic acid, which neither raises nor lowers serum cholesterol levels $(33,34)$. High protein levels in beef are beneficial for body weight management (35), sarcopenia (36) and other physiological functions (37), which are helpful in the recovery of muscles after stroke. BD treatment of rats with cerebral ischemia improved their mental state and activity, which reflects the unique advantage of dietary therapy. In rats, occlusion of the middle cerebral artery resulted in significant neurological deficiencies. According to the neurological observations of the present study, BD treatment at various doses or for various durations significantly reduced the neurological deficit scores in rats with cerebral ischemia compared to those in vehicle-treated ones. However, different doses of BD did not significantly change the deficit scores, which may be due to the small sample size and short duration of the experiment.

The infarct volume is the primary outcome for experimental studies on ischemic stroke (38) and it is the most vigorous index of ischemic brain damage (39). In the present study, BD treatment for 7 days significantly reduced the infarct volume in the pMCAO rats $(\mathrm{P}<0.05)$. In the second experiment, $\mathrm{BD}$ at doses of 400 and $600 \mathrm{mg} / \mathrm{kg}$ reduced the infarct volume after 7 days of treatment $(\mathrm{P}<0.01)$, but no difference in reduction was identified between different dosages. These results clearly demonstrated that higher dosage of $\mathrm{BD}$, i.e., 400 and $600 \mathrm{mg} / \mathrm{kg}$ for 7 days, protected the brain from ischemic injury. 
A

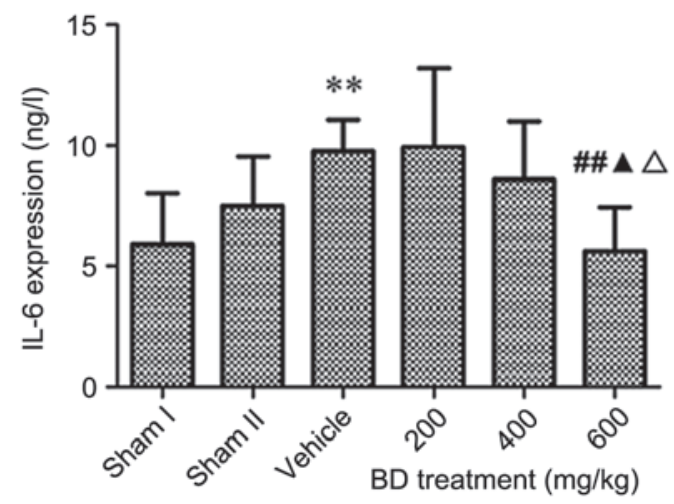

C

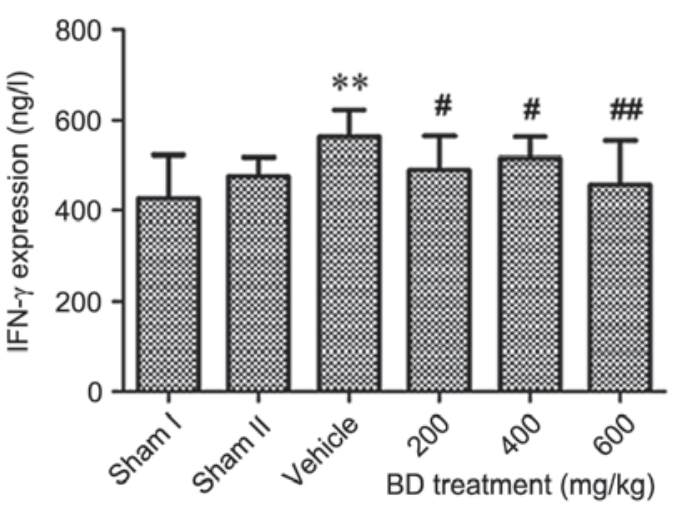

B

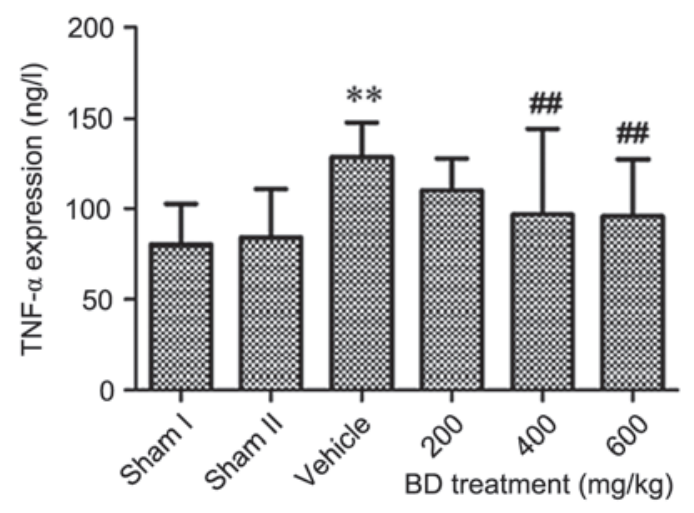

D

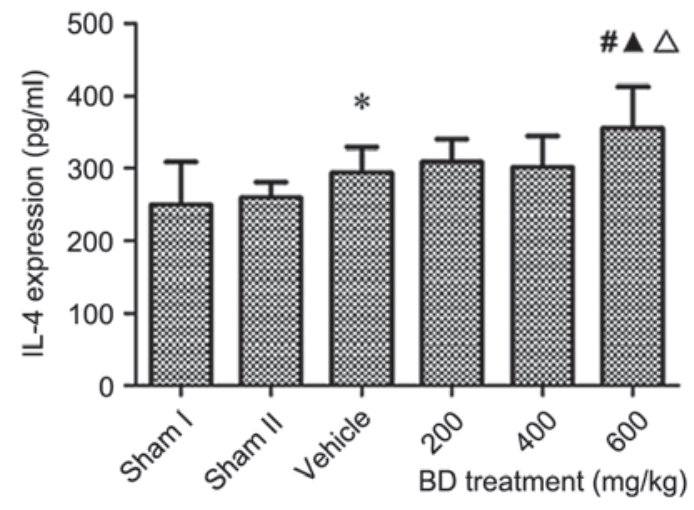

Figure 7. BD treatment affects inflammatory cytokine levels in the blood following ischemic injury. ELISA was performed to detect the levels of the pro-inflammatory cytokines (A) IL-6, (B) TNF- $\alpha$, (C) IFN- $\gamma$ and the anti-inflammatory cytokine (D) IL-4 at day 7 after treatment. ${ }^{*} \mathrm{P}<0.05$, ** P<0.01, compared with sham group; ${ }^{\#} \mathrm{P}<0.05,{ }^{\# \#} \mathrm{P}<0.01$, vs. the vehicle group; ${ }^{\mathbf{A}} \mathrm{P}<0.01$, vs. the $200 \mathrm{mg} / \mathrm{kg}$ BD-treated group; ${ }^{\Delta} \mathrm{P}<0.01$, vs. the $400 \mathrm{mg} / \mathrm{kg}$ BD-treated group. Values are expressed as the mean \pm standard deviation. The sham I group received normal saline, while the sham II group was treated with $600 \mathrm{mg} / \mathrm{kg}$ BD. BD, beef decoction; IL, interleukin; TNF, tumor necrosis factor; IFN, interferon.

Inflammation has an important role in cerebral ischemia injury. After ischemia, immune cells, microglia and macrophages are activated by different stimulants leading to the production of inflammatory cytokines, chemokines and other cytotoxic mediators, causing prolonged inflammation for several days after stroke onset (9). During this process, the balance between pro- and anti-inflammatory cytokines or $\mathrm{T}$ helper 1 (Th1) and Th2 cytokines is critical in the initiation and regulation of the immune system $(40,41)$. Th1 and Th2 lymphocytes are differentiated by $\mathrm{CD} 4^{+} \mathrm{Th} 0$ lymphocytes (42). Th1 cells mainly secrete IL-2, TNF- $\alpha$, IFN- $\gamma$ and IL-12p70, supporting cellular immunity, while Th2 cells secrete IL-4, IL-6 and IL-10, mediating humoral immunity (43).

Previous studies have reported a shift from Th1 to Th2 production after $\mathrm{pMCAO}$, and that ischemia-induced immunosuppression showed a decrease in Th1 cytokines, IL-2 and IL-12 with a significant increase in Th2 cytokines, IL-4 and IL-6 (44-46). However, in the present study, apart from the Th2 cytokines (IL-4, IL-6), the levels of Th1 cytokines (TNF- $\alpha$, IFN- $\gamma$ ) were also significantly elevated in serum of pMCAO rats after 7 days compared to those in the sham I group, which is in part consistent with the findings of a previous study (46). IL-6, TNF- $\alpha$ and IFN- $\gamma$ are pro-inflammatory cytokines in the neuropathology of cerebral ischemia $(47,48)$. Upregulation of IL-6, TNF- $\alpha$ and IFN- $\gamma$ after ischemia results in the disruption of the blood-brain barrier and an increase in the infarct volume, leading to neuronal cell death (49). In the present study, elevation of IL-6, TNF- $\alpha$ and IFN- $\gamma$ may be responsible for the ischemia-induced nerve damage.

IL-4 secreted by Th 2 cells is one of main anti-inflammatory cytokines in the inflammatory cascade reaction of cerebral ischemia. Expression of IL-4 was increased in the serum of pMCAO rats, although the difference was not significant in the present study ( $\mathrm{P}>0.05)$. Reducing the levels of pro-inflammatory cytokines and increasing the levels of anti-inflammatory cytokines is an effective way to relieve inflammatory damage after cerebral ischemia.

After BD treatment at 200, 400 and $600 \mathrm{mg} / \mathrm{kg}$, IFN- $\gamma$ levels were significantly downregulated in rats with cerebral ischemia, while IL-6 expression was only significantly downregulated after treatment with $600 \mathrm{mg} / \mathrm{kg} \mathrm{BD}(\mathrm{P}<0.01)$. Furthermore, 400 and $600 \mathrm{mg} / \mathrm{kg}$ BD markedly decreased the level of TNF- $\alpha$ $(\mathrm{P}<0.01)$. Therefore, treatment with BD at $600 \mathrm{mg} / \mathrm{kg}$ suppressed the inflammatory response by decreasing ischemia-induced pro-inflammatory cytokines. At the same time, BD treatment at the dose of $600 \mathrm{mg} / \mathrm{kg}$ increased the anti-inflammatory cytokine IL-4 $(\mathrm{P}<0.05)$. In addition, compared to the low and medium dose, the high dose of BD significantly increased the level of IL-6 $(\mathrm{P}<0.01)$, while significantly decreasing the expression of IL-4 $(\mathrm{P}<0.01)$, reflecting that the effect of $\mathrm{BD}$ 
on cytokine expression is dose-dependent. BD suppressed the inflammatory response by decreasing ischemia-induced pro-inflammatory cytokines and increasing anti-inflammatory cytokines, suggesting that BD is a powerful inflammation regulatory agent, as it contains carnosine (25). Additional mechanisms of action of $\mathrm{BD}$ decreasing brain damage require exploration in further studies. Furthermore, the in-depth mechanism of BD inducing anti-inflammatory cytokines requires to be deduced to understand the associated pathways.

The present study had certain limitations; for instance, the level of BD and its active component carnosine in the brain after the intake, which may have varied between the rats, was not assessed in the present study. Furthermore, the effect of other compounds contained in BD, which may exhibit neuroprotective effects, requires to be explored in further studies to provide more insight on the effects of BD as a whole.

In conclusion, BD enriched with carnosine showed neuroprotective effects against ischemia-induced brain damage in rats by regulating the balance between pro- and anti-inflammatory cytokines. BD may be considered as a novel supplement food for treating ischemic stroke. The molecular mechanisms and clinical applications of BD require further investigation.

\section{Acknowledgements}

This study was supported by the Projects in Humanities and Social Sciences of the Ministry of Education in China (grant no. 10YJCZH145).

\section{References}

1. Lozano R, Naghavi M, Foreman K, Lim S, Shibuya K, Aboyans V, Abraham J, Adair T, Aggarwal R, Ahn SY, et al: Global and regional mortality from 235 causes of death for 20 age groups in 1990 and 2010: A systematic analysis for the global burden of disease study 2010. Lancet 380: 2095-2128, 2012.

2. Murray CJ, Vos T, Lozano R, Naghavi M, Flaxman AD, Michaud C, Ezzati M, Shibuya K, Salomon JA, Abdalla S, et al: Disability-adjusted life years (DALYs) for 291 diseases and injuries in 21 regions, 1990-2010: A systematic analysis for the global burden of disease study 2010. Lancet 380: 2197-2223, 2012.

3. Demaerschalk BM, Hwang HM and Leung G: US cost burden of ischemic stroke: A systematic literature review. Am J Manag Care 16: 525-533, 2010.

4. Khealani BA, Khan M, Tariq M, Malik A, Siddiqi AI, Awan S and Wasay M: Ischemic strokes in Pakistan: Observations from the national acute ischemic stroke database. J Stroke Cerebrovasc Dis 23: 1640-1647, 2014.

5. Muir KW: Stroke. Medicine 41: 169-174, 2013.

6. González RG, Hirsch JA, Koroshetz WJ, Lev MH and Schaefer PW (eds): Acute Ischemic Stroke: Imaging and Intervention. Springer-Verlag, Berlin, pp41-56, 2006.

7. Zhu C, Zhang X, Qiao H, Wang L, Zhang X, Xing Y, Wang C, Dong L, Ji Y and Cao X: The intrinsic PEDF is regulated by PPARgamma in permanent focal cerebral ischemia of rat. Neurochem Res 37: 2099-2107, 2012.

8. Tuttolomondo A, Di Raimondo D, Pecoraro R, Arnao V, Pinto A and Licata G: Inflammation in ischemic stroke subtypes. Curr Pharm Des 18: 4289-4310, 2012.

9. Shichita T, Ito M and Yoshimura A: Post-ischemic inflammation regulates neural damage and protection. Front Cell Neurosci 8: $319,2014$.

10. Biancofiore G, Bindi L, Miccoli M, Metelli MR, Panicucci E, Baggiani A and Filipponi F: Balance of pro- and anti-inflammatory cytokines in cirrhotic patients undergoing liver transplantation. Transpl Immunol 28: 193-197, 2013.

11. Wang B, Sun JN, Sun WY and Zhang SF: Effects of Qingnaoxuanqiao Droping Pill on inflammatory factor in brain of acute cerebral ischemia-reperfusion injury rat. Pharmacol Clin Chin Mater Med 26: 112-114, 2010 (In Chinese).
12. Crush KG: Carnosine and related substances in animal tissues. Comp Biochem Physiol 34: 3-30, 1970.

13. Rajanikant GK, Zemke D, Senut MC, Frenkel MB, Chen AF, Gupta R and Majid A: Carnosine is neuroprotective against permanent focal cerebral ischemia in mice. Stroke 38: 3023-3031, 2007.

14. Bae ON, Serfozo K, Baek SH, Lee KY, Dorrance A, Rumbeiha W, Fitzgerald SD, Farooq MU, Naravelta B, Bhatt A and Majid A: Safety and efficacy evaluation of carnosine, an endogenous neuroprotective agent for ischemic stroke. Stroke 44: 205-212, 2013.

15. Shen Y, He P, Fan YY, Zhang JX, Yan HJ, Hu WW, Ohtsu H and Chen Z: Carnosine protects against permanent cerebral ischemia in histidine decarboxylase knockout mice by reducing glutamate excitotoxicity. Free Radic Biol Med 48: 727-735, 2010.

16. Wang JP, Yang ZT, Liu C, He YH and Zhao SS: L-carnosine inhibits neuronal cell apoptosis through signal transducer and activator of transcription 3 signaling pathway after acute focal cerebral ischemia. Brain Res 1507: 125-133, 2013.

17. Wang AH, Zhu J, Liu CM and Xu GH: Effects of carnosine on T-lymphocyte immunity in peripheral blood of rats with cerebral ischemia. Acta Univ Med Nanjing (Nat Sci) 34: 1333-1336, 2014 (In Chinese).

18. Ma Q, Zhu J, Wang X and Wang AH: Effect of beef decoction on levels of serum interleukin- 6 and tumor necrosis factor- $\alpha$ in rats of cerebral infraction. Chin Neuroimmunol Neuro 22: 62-66, 2015.

19. Longa EZ, Weinstein PR, Carlson S and Cummins R: Reversible middle cerebral artery occlusion without craniectomy in rats. Stroke 20: 84-91, 1989.

20. Lee TH, Jung CH and Lee DH: Neuroprotective effects of Schisandrin B against transient focal cerebral ischemia in Sprague-Dawley rats. Food Chem Toxicol 50: 4239-4245, 2012.

21. De Marchis S, Modena C, Peretto P, Migheli A, Margolis FL and Fasolo A: Carnosine-related dipeptides in neurons and glia. Biochemistry (Mosc) 65: 824-833, 2000.

22. Dziennis S and Alkayed NJ: Role of signal transducer and activator of transcription 3 in neuronal survival and regeneration. Rev Neurosci 19: 341-361, 2008.

23. Jin CL, Yang LX, Wu XH, Li Q, Ding MP, Fan YY, Zhang WP, Luo JH and Chen Z: Effects of carnosine on amygdaloid-kindled seizures in Sprague-Dawley rats. Neuroscience 135: 939-947, 2005.

24. Pekcetin C, Kiray M, Ergur BU, Tugyan K, Bagriyanik HA, Erbil G, Baykara B and Camsari UM: Carnosine attenuates oxidative stress and apoptosis in transient cerebral ischemia in rats. Acta Biol Hung 60: 137-148, 2009.

25. Fan FY, Huang LZ, Wang GY and Zhou H: Effect of carnosine on the expression of HSP70 and inflammatory factors in a rat ischemia-reperfusion injury model. J Med Mol Biol 4: 11-14, 2007.

26. Zhang XM, Zhang Z, Cheng XY and Pediatrics DO: The effect of carnosine on apoptosis biochemical markers of HI rats. Chin Pediatr Integr Tradit West Med 6: 2014 (In Chinese).

27. Rinderknecht H, Rebane T and Ma V: Synthesis of carnosine, anserine and isoanserine. J Org Chem 39: 1968-1970, 1964.

28. Sharif S, Schagen D, Toney MD and Limbach HH: Coupling of functional hydrogen bonds in pyridoxal-5'-phosphate-enzyme model systems observed by solid-state NMR spectroscopy. J Am Chem Soc 129: 4440-4455, 2007.

29. Gopalakrishnan J, Decker EA and Means WJ: Antioxidant activity of mechanically separated pork extracts. Meat Sci 52: 101-110, 1999.

30. O'Neil CE, Zanovec M, Keast DR, Fulgoni VL III and Nicklas TA: Nutrient contribution of total and lean beef in diets of US children and adolescents: National health and nutrition examination survey 1999-2004. Meat Sci 87: 250-256, 2011.

31. Zanovec M, O'Neil CE, Keast DR, Fulgoni VL III and Nicklas TA: Lean beef contributes significant amounts of key nutrients to the diets of US adults: National health and nutrition examination survey 1999-2004. Nutr Res 30: 375-381, 2010.

32. Nicklas TA, O'Neil CE, Zanovec M, Keast DR and Fulgoni VL III: Contribution of beef consumption to nutrient intake, diet quality and food patterns in the diets of the US population. Meat Sci 90: 152-158, 2012.

33. Daley CA, Abbott A, Doyle PS, Nader GA and Larson S: A review of fatty acid profiles and antioxidant content in grass-fed and grain-fed beef. Nutr J 9: 10, 2010.

34. Hunter JE, Zhang J and Kris-Etherton PM: Cardiovascular disease risk of dietary stearic acid compared with trans, other saturated, and unsaturated fatty acids: A systematic review. Am J Clin Nutr 91: 46-63, 2010. 
35. Paddon-Jones D, Westman E, Mattes RD, Wolfe RR, Astrup A and Westerterp-Plantenga M: Protein, weight management, and satiety. Am J Clin Nutr 87: 1558S-1561S, 2008.

36. Paddon-Jones D, Short KR, Campbell WW, Volpi E and Wolfe RR: Role of dietary protein in the sarcopenia of aging. Am J Clin Nutr 87: 1562S-1566S, 2008.

37. Wolfe RR, Miller SL and Miller KB: Optimal protein intake in the elderly. Clin Nutr 27: 675-684, 2008.

38. McBride DW, Klebe D, Tang J and Zhang JH: Correcting for brain swelling's effects on infarct volume calculation after middle cerebral artery occlusion in rats. Transl Stroke Res 6: 323-338, 2015

39. Liang H, Liu P, Wang Y, Song S and Ji A: Protective effects of alkaloid extract from Leonurus heterophyllus on cerebral ischemia reperfusion injury by middle cerebral ischemic injury (MCAO) in rats. Phytomedicine 18: 811-818, 2011.

40. London CA, Abbas AK and Kelso A: Helper T cell subsets: Heterogeneity, functions and development. Vet Immunol Immunopathol 63: 37-44, 1998.

41. Gonzalez-Rey E, Chorny A and Delgado M: Regulation of immune tolerance by anti-inflammatory neuropeptides. Nat Rev Immunol 7: 52-63, 2007.

42. Poittevin M, Deroide N, Azibani F, Delcayre C, Giannesini C, Levy BI, Pocard M and Kubis N: Glatiramer Acetate administration does not reduce damage after cerebral ischemia in mice. J Neuroimmunol 254: 55-62, 2013.

43. Gee JM, Kalil A, Shea C and Becker KJ: Lymphocytes: Potential mediators of postischemic injury and neuroprotection. Stroke 38 (2 Suppl): S783-S788, 2007.
44. Kim HM, Shin HY, Jeong HJ, An HJ, Kim NS, Chae HJ, Kim HR, Song HJ, Kim KY, Baek SH, et al: Reduced IL-2 but elevated IL-4, IL-6, and IgE serum levels in patients with cerebral infarction during the acute stage. J Mol Neurosci 14: 191-196, 2000.

45. Song B, Tang X, Wang X, Huang X, Ye Y, Lu X, Wei X and Zeng Y: Bererine induces peripheral lymphocytes immune regulations to realize its neuroprotective effects in the cerebral ischemia/reperfusion mice. Cell Immunol 276: 91-100, 2012.

46. Hu T, Fu Q, Liu X, Zhang $\mathrm{H}$ and Dong M: Increased acetylcholinesterase and capase-3 expression in the brain and peripheral immune system of focal cerebral ischemic rats. J Neuroimmunol 211: 84-91, 2009.

47. Chio CC, Lin JW, Chang MW, Wang CC, Kuo JR, Yang CZ and Chang CP: Therapeutic evaluation of etanercept in a model of traumatic brain injury. J Neurochem 115: 921-929, 2010.

48. Zeng L, Wang Y, Liu J, Wang L, Weng S, Chen K, Domino EF and Yang GY: Pro-inflammatory cytokine network in peripheral inflammation response to cerebral ischemia. Neurosci Lett 548: 4-9, 2013.

49. Lambertsen KL, Biber K and Finsen B: Inflammatory cytokines in experimental and human stroke. J Cereb Blood Flow Metab 32: $1677-1698,2012$

(i) $\Theta$ This work is licensed under a Creative Commons Attribution-NonCommercial-NoDerivatives 4.0 International (CC BY-NC-ND 4.0) License. 